Supporting Information

\title{
Atomic Plane-Vacancy Engineering of
}

\section{Transition Metal Dichalcogenides with}

\section{Enhanced Hydrogen Evolution Capability}

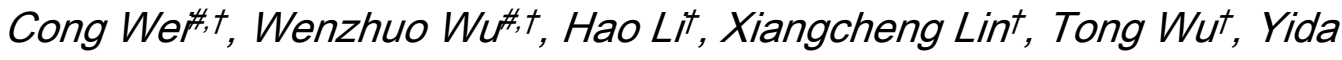

Zhang ${ }^{\ddagger}$, Quan Xu*:, Lipeng Zhangs, Yonghao Zhus, Xinan Yangll, Zheng Liu',

Qun $X u^{*}+t$

${ }^{\dagger}$ College of Materials Science and Engineering, Zhengzhou University,

Zhengzhou 450001, China, E-mail: qunxu@zzu.edu.cn;

‡State Key Laboratory of Petroleum Resources and Prospecting, Harvard

SEAS-CUPB Joint Laboratory on Petroleum Science, 29 Oxford Street, Cambridge, MA 02138, USA;

§College of Engineering, Beijing University of Chemical Technology, Beijing, 100029, China;

\|National Laboratory for Condensed Matter Physics and Institute of Physics, Chinese Academy of Sciences, Beijing, China;

${ }^{\perp}$ School of Materials Science and Engineering, Nanyang Technological University, Singapore 639798, Singapore. 
\#Dr. C. Wei and W. Z. Wu contributed equally to this work. 


\section{Contents}

1. Materials and Characterizations.

1). Materials.

2). Characterizations.

2. Theoretical calculations.

3. Figure S1. SEM images of bulk-MoS 2 before and after $\mathrm{NaBH}_{4}$ treatment.

4. Figure S2. XRD pattern of annealed bulk $\mathrm{MoS}_{2}, \mathrm{NaBH}_{4}$ and their mixture.

5. Figure S3. XRD pattern analysis of peaks shift in annealed $\mathrm{MoS}_{2}$.

6. Figure S4. Yields of Sv-MoS 2 nanosheets by using SPR method.

7. Figure S5. TEM images of ultrasonic exfoliated $\mathrm{MoS}_{2} \mathrm{NSs}$ and Sv-MoS 2 NSs prepared via SPR strategy.

8. Figure S6. XRD pattern of bulk $\mathrm{MoS}_{2}$ and $\mathrm{Sv}-\mathrm{MoS}_{2} \mathrm{NSs}$.

9. Figure S7. High-resolution STEM-ADF image of few layer $2 \mathrm{H}-\mathrm{MoS}_{2}$.

10. Figure S8. XPS spectra of P-MoS 2 NSs and Sv-MoS 2 NSs.

11. Figure S9. Raman spectra for the ultrasonic exfoliated 2D-MoS ${ }_{2}$ and SPR produced Sv-MoS 2 NSs.

12. Figure S10. $J-V$ curves of pristine bulk-MoS 2 and $\mathrm{NaBH}_{4}$ treated bulk-MoS 2 .

13. Figure S11. Cyclic voltammetry curves of the Sv-MoS 2 NSs.

14. Figure S12. Band structure of monolayer $2 \mathrm{H}-\mathrm{MoS}_{2}$ with and without Svacancies.

15. Figure S13. XRD pattern of annealed bulk $\mathrm{WS}_{2}, \mathrm{NaBH}_{4}$ and their mixture.

16. Figure S14. XPS spectra of P-WS ${ }_{2} \mathrm{NSs}$ and $\mathrm{Sv}-\mathrm{WS}_{2} \mathrm{NSs}$. 
17. Figure $\mathrm{S} 15 . \mathrm{J}-\mathrm{V}$ curves of pristine bulk-WS$S_{2}$ and $\mathrm{NaBH}_{4}$ treated bulk-WS $\mathrm{S}_{2}$.

18. Figure S16. Cyclic voltammetry curves of Sv-WS $\mathrm{WSs}_{2}$

19. Table S1. Summary of strategies for S vacancy in $\mathrm{MoS}_{2}$, and their HER activities in $0.5 \mathrm{M} \mathrm{H}_{2} \mathrm{SO}_{4}$.

20. Table S2. The free energy of different models and the length of bong between the active center and hydrogen atom.

21. Table S3. Summary of HER activities of Sv-WS 2 and the reported $\mathrm{WS}_{2}$ materials in $0.5 \mathrm{M} \mathrm{H}_{2} \mathrm{SO}_{4}$.

\section{Materials and Characterizations.}

1). Materials.

The $\mathrm{MoS}_{2}$ and $\mathrm{WS}_{2}$ powder were purchased from Sigma-Aldrich. $\mathrm{NaBH}_{4}, \mathrm{HCl}$ and ethanol used were purchased from Sinopharm Chemical Reagent Co., Ltd. (China) and used without further treatment. Nitogen with a purity of $99.99 \%$ was provided by the Zhengzhou Shuangyang Gas Co. and used as received.

2). Characterizations.

The morphology of the samples was measured with transmission electron microscopy (TEM) (JEM-2100) and high resolution TEM (HRTEM, $200 \mathrm{kV}$ or $300 \mathrm{kV}$ ). The aberration-corrected scanning transmission electron microscopy (STEM) images of samples were measured with JEOL JEM-ARM300F. The 
Raman tests of samples were carried out on a laser micro-Raman spectrometer (Renishaw inVia) equipped with a $50 \mathrm{~mW} \mathrm{Ar}{ }^{+}$laser (wavelength $=514.5 \mathrm{~nm}$ ). The powder X-ray diffraction (XRD) patterns of samples were recorded on a Rigaku D/Max 2550 X-ray diffractometer with Cu Ka radiation $(\lambda=1.5418 \AA$ ). Interlamellar spacing (d) of samples were calculated by

$$
2 d \sin \theta=\mathrm{n} \lambda
$$

The X-ray photoelectron spectroscopy (XPS) spectra of samples were performed on an XPS, ESCA Lab250 X-ray photoelectron spectrometer. All XPS spectra were corrected using C 1s line at $284.6 \mathrm{eV}$, and curve fitting and background subtraction were accomplished.

\section{Theoretical calculations.}

First-principle calculations were carried out by DFT using the plane wave set VASP code with the Perdew-Burke-Ernzerhof (PBE) exchange-correlation. The plane basis set has a cutoff energy of $500 \mathrm{eV}$ throughout the computations. All calculations were spin polarized and were done until the force of the system converges to about $0.01 \mathrm{eV} / \mathrm{A}$. The convergence criterion for electronic structure iteration was set as $10^{-4} \mathrm{eV}$. MoS 2 structures were built with periodical boundary in $\mathrm{x}, \mathrm{y}$ directions, and in $\mathrm{z}$ direction, a vacuum space was added with the value of $20 \AA$. The k-point sampling of the Brillouin zone was obtained using a $6 \times 6 \times 1$ grid generating meshes with their origin centered at the gamma $(\Gamma)$ point. 
The Gibbs free energy of the adsorption of atomic hydrogen is obtained as,

$$
\Delta G_{H^{*}}=\Delta E_{H}+\Delta E_{Z P E}-T \Delta S_{H}
$$

where $\Delta E_{H}, \Delta E_{Z P E}, \Delta S_{H}$ represent hydrogen adsorption energy, the change of zero point energy, and difference entropy between the adsorption of hydrogen atom and hydrogen in the gas, respectively. The entropy of adsorption hydrogen is ignored because of huge distinction with gas hydrogen, $\Delta S_{H} \cong-1 / 2 S_{H_{2}}$. The adsorption energy (same calculation with change of zero point energy) is defined as,

$$
\Delta E_{H}=E_{H^{*}}-E_{*}-1 / 2 E_{H_{2}}
$$

where * denotes the adsorption on the surface of catalyst, $E_{H^{*}}$ and $E_{H_{2}}$ represent total energies of slab with a hydrogen atom and gas hydrogen, respectively. Therefore, the Gibbs free energy can be written as,

$$
\Delta G_{H^{*}}=\Delta E_{H}+0.24
$$

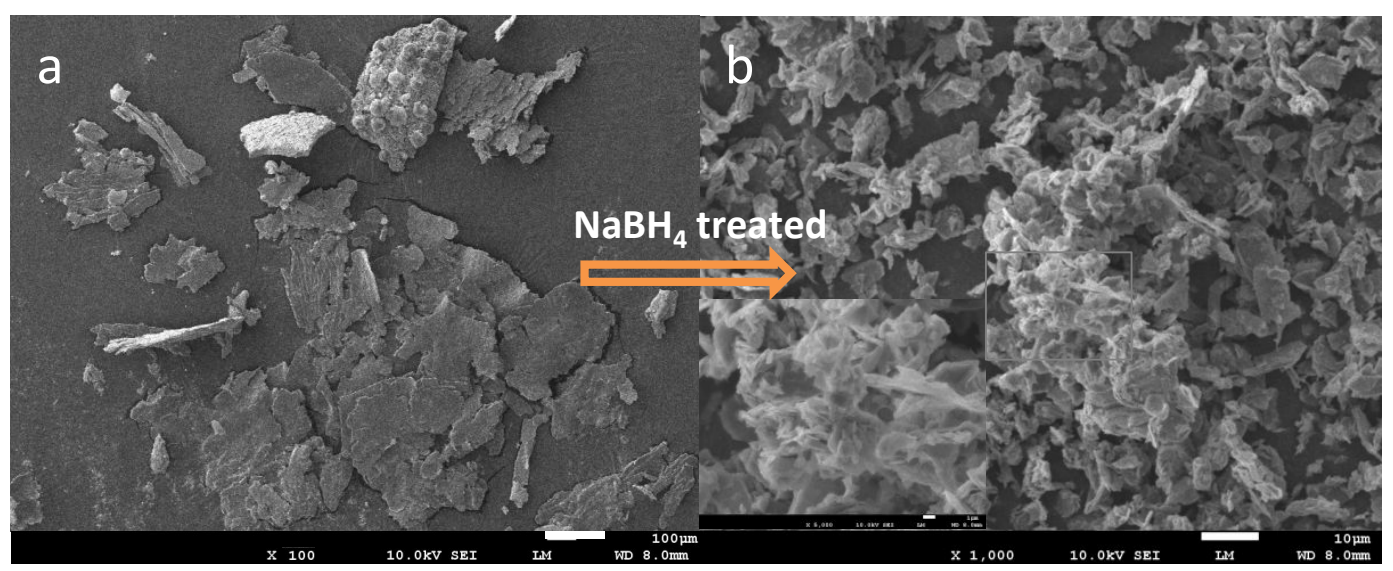

Figure S1. SEM images of bulk-MoS 2 before (a) and after (b) $\mathrm{NaBH}_{4}$ treatment. 


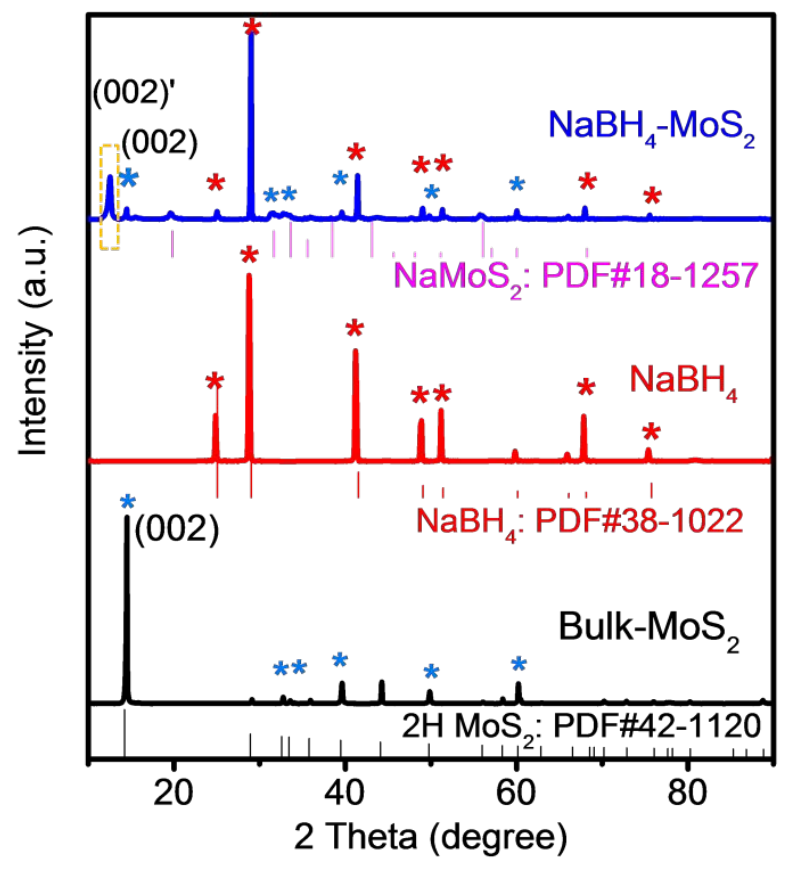

Figure S2. XRD pattern of annealed bulk $\mathrm{MoS}_{2}$ (black), $\mathrm{NaBH}_{4}$ (red) and $\mathrm{MoS}_{2} / \mathrm{NaBH}_{4}$ mixture (blue).

As shown in Figure S2 (blue), the presented peaks of the annealed mixture manifest the mixture consist of residual $\mathrm{NaBH}_{4}$ (red star), $\mathrm{MoS}_{2}$ (blue star) and $\mathrm{Na}^{+}$intercalated $\mathrm{MoS}_{2}$ compound (PDF\#18-1257). ${ }^{1}$ Notably, after SPR treatment with $\mathrm{NaBH}_{4}$, (002)' peak located at lower angle than (002) peak appeared, however, no peaks position shift occurred in pure $\mathrm{MoS}_{2}$ after being annealed without $\mathrm{NaBH}_{4}$ (Figure S2 black), demonstrating that addition of $\mathrm{NaBH}_{4}$ is responsible for the expansion of $\mathrm{MoS}_{2}$ during the SPR process. 


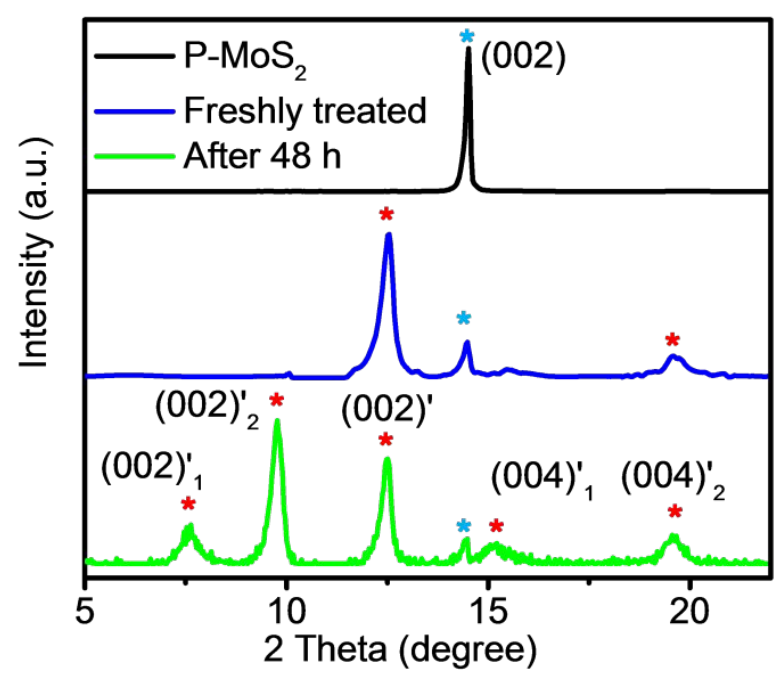

Figure S3. XRD patterns for samples of P-MoS 2 (black), freshly SPR treated $\mathrm{MoS}_{2}$ (blue) and SPR treated $\mathrm{MoS}_{2}$ after being exposed to the ambient atmosphere for $48 \mathrm{~h}$ (green). 


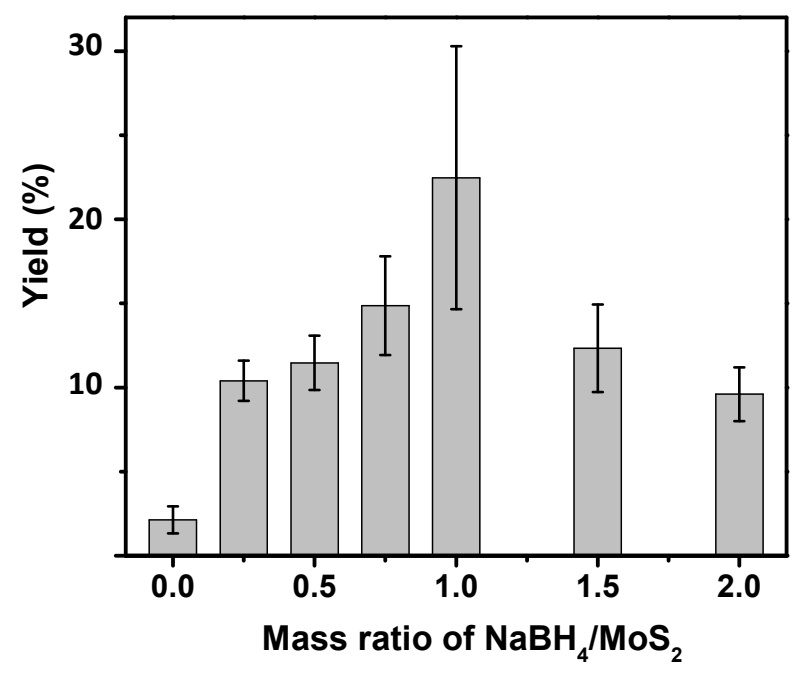

Figure S4. Yields of $\mathrm{MoS}_{2}$ nanosheets prepared from bulk $\mathrm{MoS}_{2}$ by using our SPR method with various dosage of $\mathrm{NaBH}_{4}$.

The mass yield of S-vacancy-MoS ${ }_{2}$ nanosheets was calculated as the weight of the obtained nanosheets divided by the weight of the used bulk $\mathrm{MoS}_{2}$. The unexfoliated $\mathrm{MoS}_{2}$ were removed by centrifugation (5000 rpm; $30 \mathrm{~min}$ ) and the exfoliated 2D-MoS 2 nanosheets were obtained by centrifuging the remaining supernatant at $20000 \mathrm{rpm}$ for 10 min. During thermal treatment with $\mathrm{NaBH}_{4}$, sodium ions would diffuse and insert into the interlayer spaces of the $\mathrm{MoS}_{2}$, gaining distinct interlayer expansion of 16\% (Figure 1c), which would increase the interlamellar spacing and promote the exfoliation of $\mathrm{MoS}_{2}$ nanosheets during the liquid phase treatment process. Thus, the mass yield of S-vacancy$\mathrm{MoS}_{2}$ nanosheets can be increased up to $24 \%$ (Figure S4). 


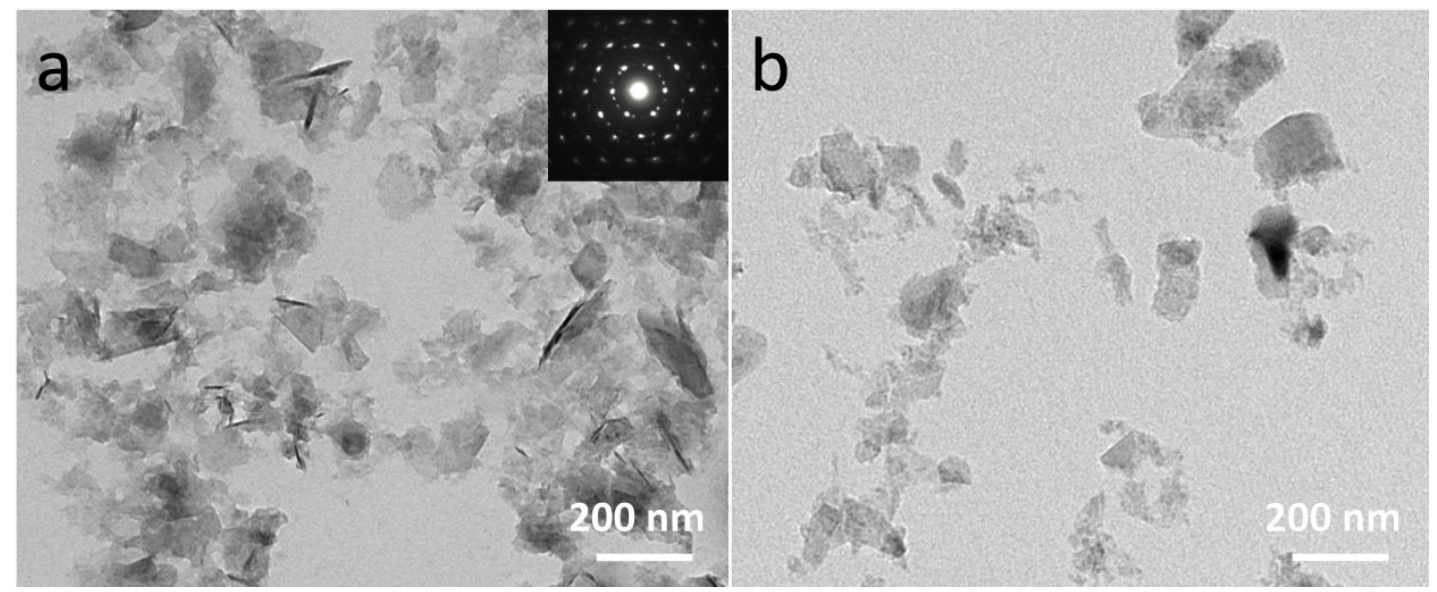

Figure S5. TEM images of Sv-MoS 2 NSs prepared via SPR strategy (a) and ultrasonic exfoliated $\mathrm{MoS}_{2}$ NSs (b).

As shown in Figure S5a, clusters of as-prepared Sv-MoS ${ }_{2} \mathrm{NSs}$ were found, the integral lateral size ranged from 100 to $200 \mathrm{~nm}$. The corresponding selectedarea electron diffraction (SAED) pattern (Figure S5a, inset) showed distinct sixfold symmetry diffraction spots, revealing the well crystallinity and hexagonal lattice of SPR treated $\mathrm{MoS}_{2}{ }^{3}$

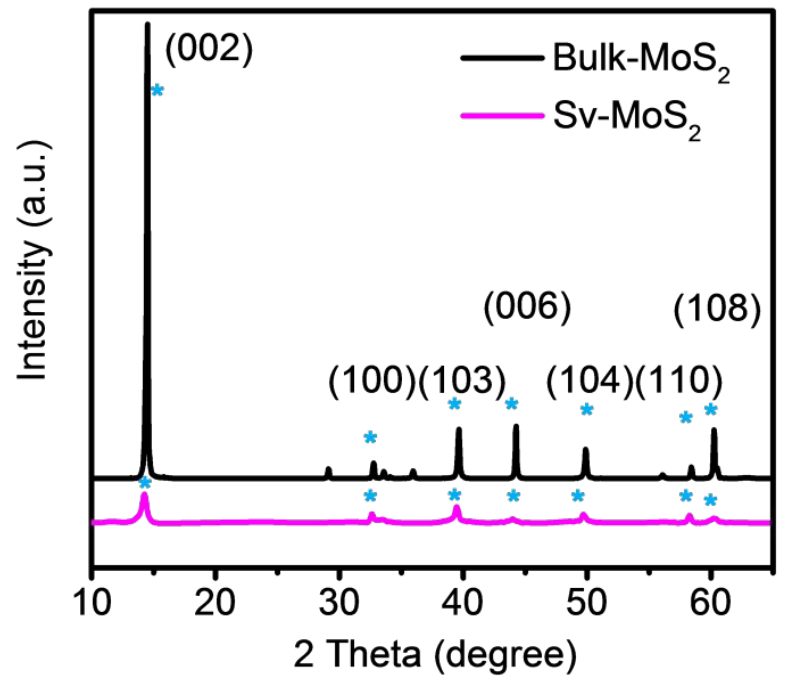

Figure S6. XRD pattern of bulk $\mathrm{MoS}_{2}$ and $\mathrm{Sv}-\mathrm{MoS}_{2} \mathrm{NSs}$. 
As shown in Figure S6, XRD pattern of the as-prepared Sv-MoS ${ }_{2}$ NSs was wellmatched with $2 \mathrm{H}-\mathrm{MoS}_{2}$. The intensity of dominant (002) peak originating from the hexagonal $2 \mathrm{H}-\mathrm{MoS}_{2}$ was used to judge the thickness of the NSs. ${ }^{4}$ Notably, the dominant decreasing peak related to the (002) plane (PDF\#37-1492) demonstrates that the Sv-MoS 2 NSs were successfully exfoliated in atomic layer thickness.
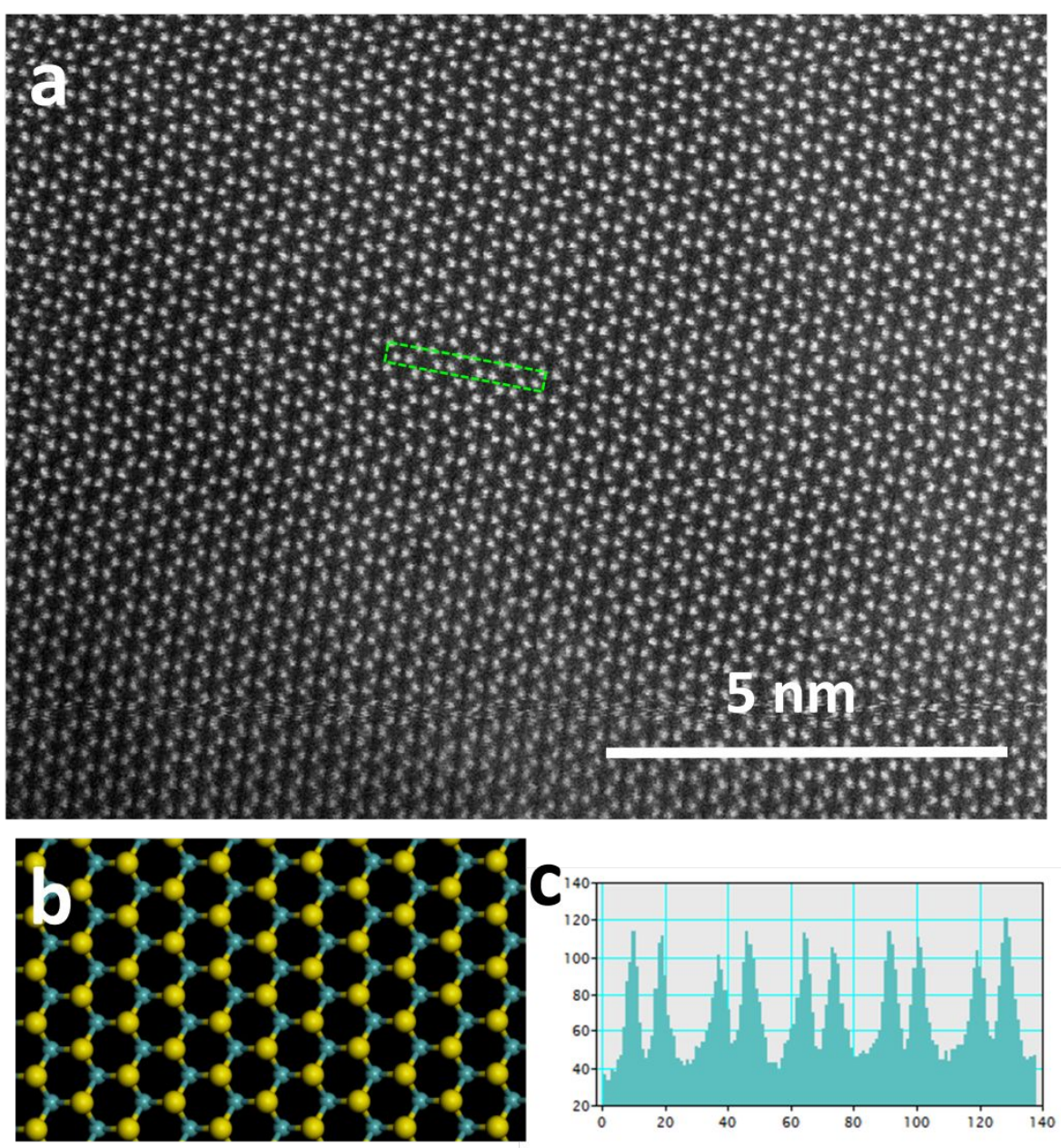
Figure S7. (a) High-resolution STEM-ADF images of the few layer $\mathrm{MoS}_{2}$ NSs.

(b) Atomic model of $2 \mathrm{H}-\mathrm{MoS}_{2}$. (c) Intensity profile of the selected line (bright cyan) in (a).
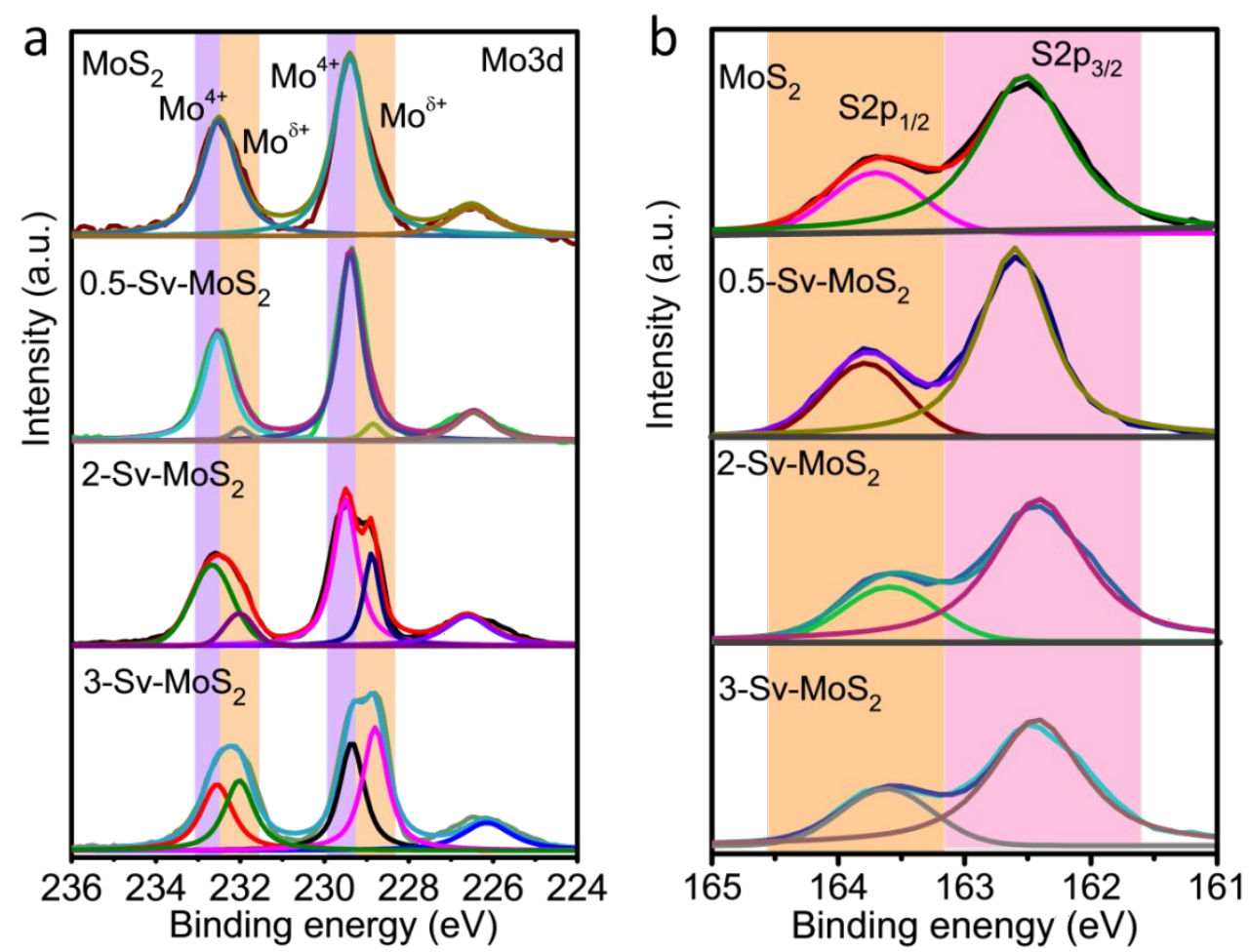

Figure S8. XPS spectra showing Mo 3d (a), S 2p (b) core level peak regions for P-MoS 2 NSs, Sv-MoS 2 NSs prepared with the mass ratio of $\mathrm{NaBH}_{4}: \mathrm{MoS}_{2}$ was $0.5,2,3$ respectively.

To investigate the sulfur depletion, XPS measurements were performed. The normalized spectra of the Mo $3 d$ state and S $2 p$ state are shown in Figure S8. The area ratios of S $2 p$ and Mo 3d peaks decomposed from P-MoS 2 NSs were used as a reference to normalize the XPS peaks, in which the value of S:Mo ratio was set as 2.0. As shown in Figure S8a, two additional Mo 3d peaks 
located at $\sim 228.9$ and $232.0 \mathrm{eV}$ were observed, and their integral areas changed with the mass ratio of $\mathrm{NaBH}_{4}: \mathrm{MoS}_{2}$ in SPR treatment, suggesting the formation of lower oxidation state, $\mathrm{Mo}^{\delta+}(\delta<4) .{ }^{5}$ In Figure S8b, the S $2 p$ peak intensity also decrease with increasing mass ratio of $\mathrm{NaBH}_{4}$ : $\mathrm{MoS}_{2}$ in SPR treatment, which is consistent with the occurrence of $\mathrm{Mo}^{\delta+}$, indicating the formation of S-vacancy.

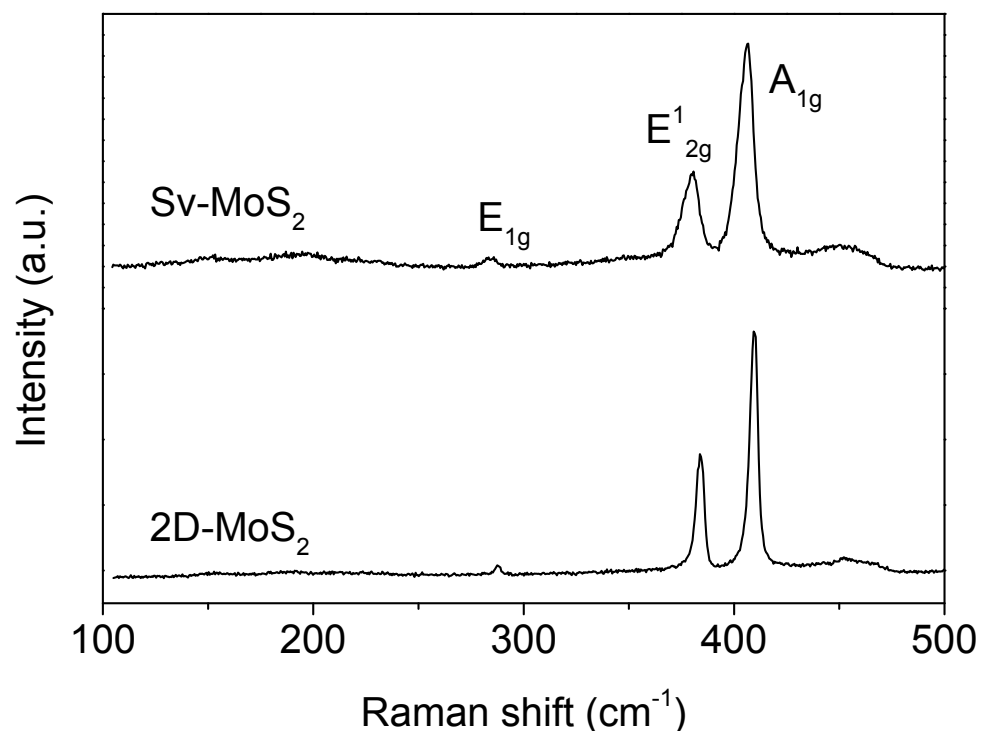

Figure S9. Raman spectra for the ultrasonic exfoliated $2 \mathrm{D}-\mathrm{MoS}_{2}$ and SPR produced $\mathrm{Sv}-\mathrm{MoS}_{2} \mathrm{NSs}$. 


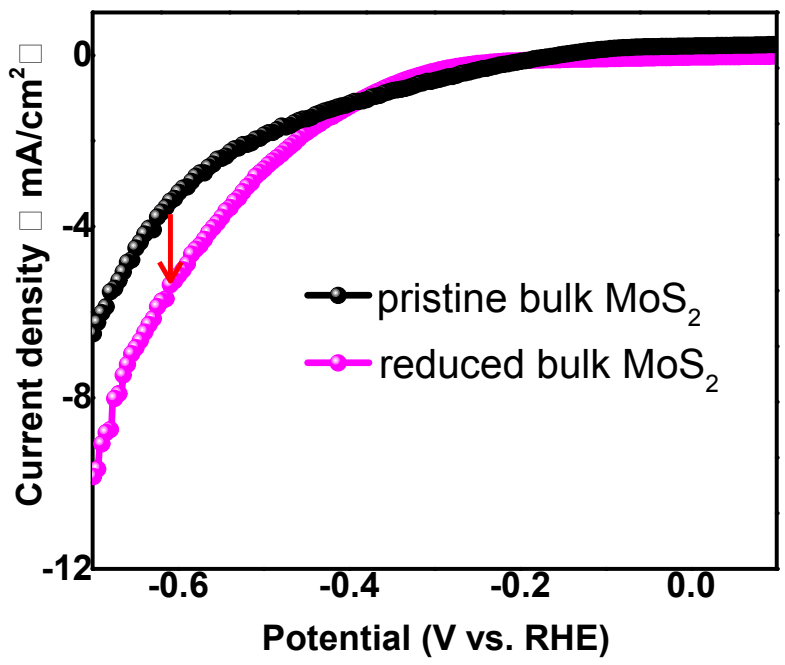

Figure S10. $\mathcal{J}-\mathrm{V}$ curves of pristine bulk-MoS and $\mathrm{NaBH}_{4}$ treated bulk-MoS 2 . 
Table S1. Summary of strategies for S vacancy in $\mathrm{MoS}_{2}$, and their HER activities in $0.5 \mathrm{M} \mathrm{H}_{2} \mathrm{SO}_{4}$.

\begin{tabular}{|c|c|c|c|c|c|}
\hline $\begin{array}{l}\text { Strategy for } \\
\text { S vacancy }\end{array}$ & $\begin{array}{l}\text { Pristine } \\
\operatorname{MoS}_{2}{ }^{a}\end{array}$ & $\begin{array}{l}\text { Catalytic } \\
\text { active } \\
\text { sites }\end{array}$ & $\eta_{-10}^{b}$ & 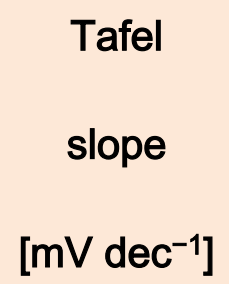 & Ref \\
\hline $\begin{array}{c}\quad \mathrm{H}_{2} \\
\text { treatment }\end{array}$ & CVD & $\begin{array}{l}\text { edge sites, } \\
\text { S vacancies, } \\
\text { grain } \\
\text { boundaries }\end{array}$ & 170 & 65 & [7] \\
\hline $\begin{array}{l}\mathrm{O}_{2} \text { plasma } \\
\text { and } \mathrm{H}_{2} \\
\text { treatment }\end{array}$ & CVD & $\begin{array}{l}\text { edge sites } \\
\text { and } \\
\text { S vacancies }\end{array}$ & $\begin{array}{l}\sim 600 \\
>600\end{array}$ & 147 & [8] \\
\hline $\begin{array}{l}\text { Electroche } \\
\text {-mical } \\
\text { generation }\end{array}$ & CVD & S vacancies & $\sim 320$ & 102 & [9] \\
\hline $\begin{array}{c}\text { Ar plasma } \\
\text { Strain }\end{array}$ & CVD & $\begin{array}{l}\text { S vacancies } \\
\text { S vacancies } \\
\text { (strained) }\end{array}$ & $\begin{array}{l}250 \\
170\end{array}$ & $\begin{array}{l}82 \\
60\end{array}$ & [10] \\
\hline SPR & $\begin{array}{l}\text { Commerci } \\
\text { al }\end{array}$ & $S$ vacancies & 238 & 64 & $\begin{array}{l}\text { This } \\
\text { work }\end{array}$ \\
\hline
\end{tabular}


Bulk $\mathrm{MoS}_{2}$

powder

a The materials which were directly used to create $S$ vacancies.

${ }^{b} \eta$ (vs. RHE) for $\mathrm{j}=-10 \mathrm{~mA} \mathrm{~cm}{ }^{-2}[\mathrm{mV}]$

Table S1 compares the HER activity of as-prepared Sv-MoS ${ }_{2}$ NSs versus various forms of Sv-MoS 2 reported in the literature.$^{6}$ The chemical composition affects the HER activity significantly. For fair comparison, Table S1 only summarizes the HER activity of $\mathrm{S}$ vacancies existed $\mathrm{MoS}_{2}$ without any heteroatomic doping. ${ }^{7-10}$ The table shows that our Sv-MoS 2 is approaching the lowest over potential at current density of $10 \mathrm{~mA} \mathrm{~cm}^{-2}$. Most importantly, only in this work the commercial bulk $\mathrm{MoS}_{2}$ is used in as initial $\mathrm{MoS}_{2}$ template for S vacancy. 
a
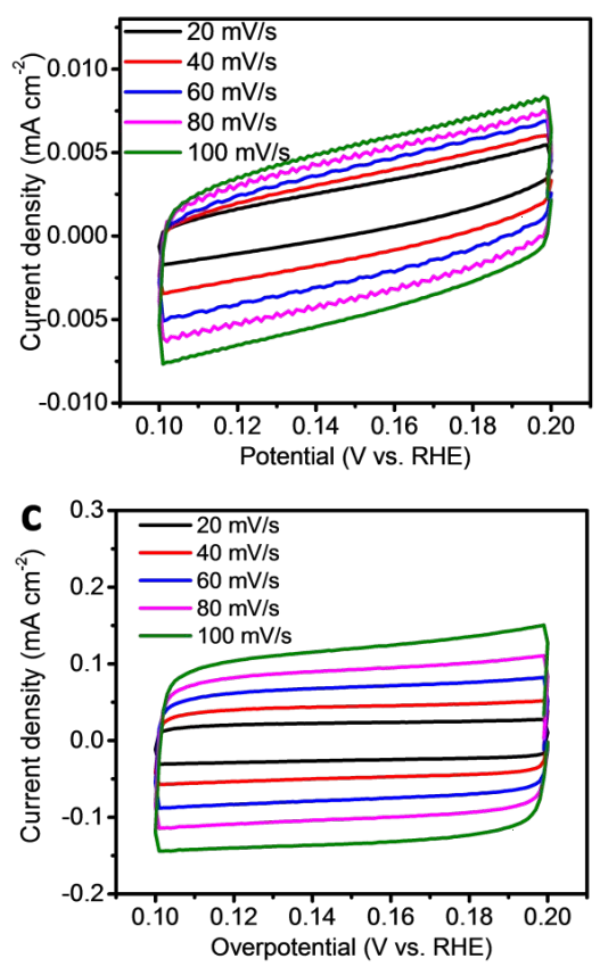

e

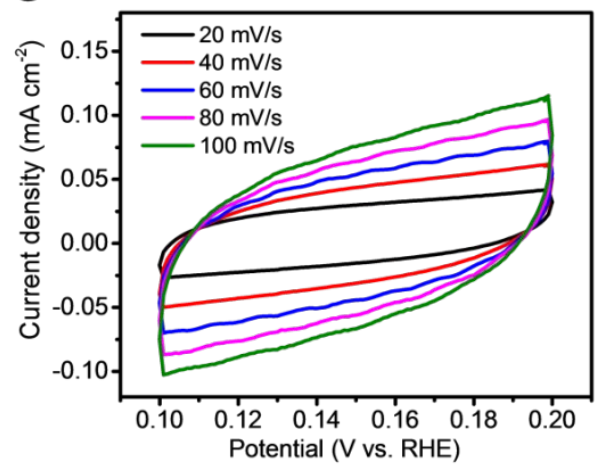

b
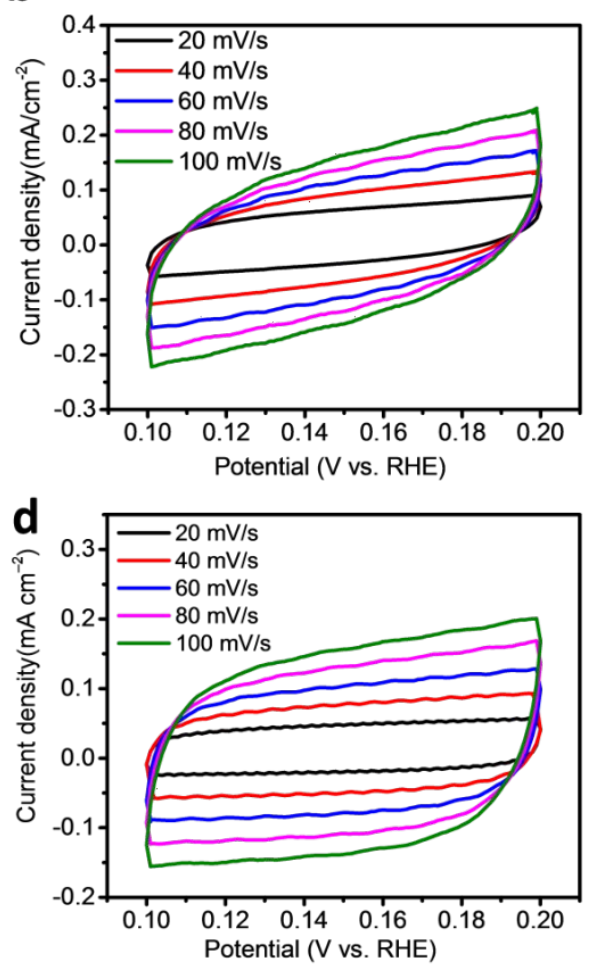

Figure S11. Cyclic voltammetry curves of (a) $\mathrm{P}-\mathrm{MoS}_{2}$, (b) $0.5-\mathrm{Sv}-\mathrm{MoS}_{2}$, (c) 1Sv-MoS 2 , (d) 2-Sv-MoS 2 NSs, (e) 3-Sv-MoS 2 NSs, respectively, under different scan rates, in the region of 0.1-0.2 V vs. RHE. These data were used to calculate the electrochemical double-layer capacitance $\left(C_{d l}\right)$. Plots show the extraction of the $C_{\mathrm{dl}}$ for different $\mathrm{MoS}_{2}$ samples shown in Figure $4 \mathrm{~d}$ in the main text. 


\begin{tabular}{|c|c|c|c|}
\hline S vacancy & Active sites & $\begin{array}{c}\text { Gibbs free energy } \\
\text { leV }\end{array}$ & $\begin{array}{l}\text { Length of bond } \\
\qquad / \AA\end{array}$ \\
\hline No-vacancy & $S$ & 1.75 & 1.40 \\
\hline \multirow{3}{*}{ One-vacancy } & $\mathrm{S}$ & 1.29 & 1.37 \\
\hline & & & \\
\hline & Mo & -0.04 & 2.00 \\
\hline \multirow{3}{*}{ Two-vacancya } & $\mathrm{S}$ & 1.18 & 1.37 \\
\hline & & & \\
\hline & Mo & -0.12 & 2.02 \\
\hline \multirow{3}{*}{ Two-vacancy } & $S$ & 1.52 & 1.36 \\
\hline & & & \\
\hline & Mo & 0.06 & 1.95 \\
\hline
\end{tabular}

Table S2. The free energy of different models and the length of bong between the active center and hydrogen atom.

a Two neighbouring $S$ vacancies in one side of plane.

b Two face-to-face S vacancies. 

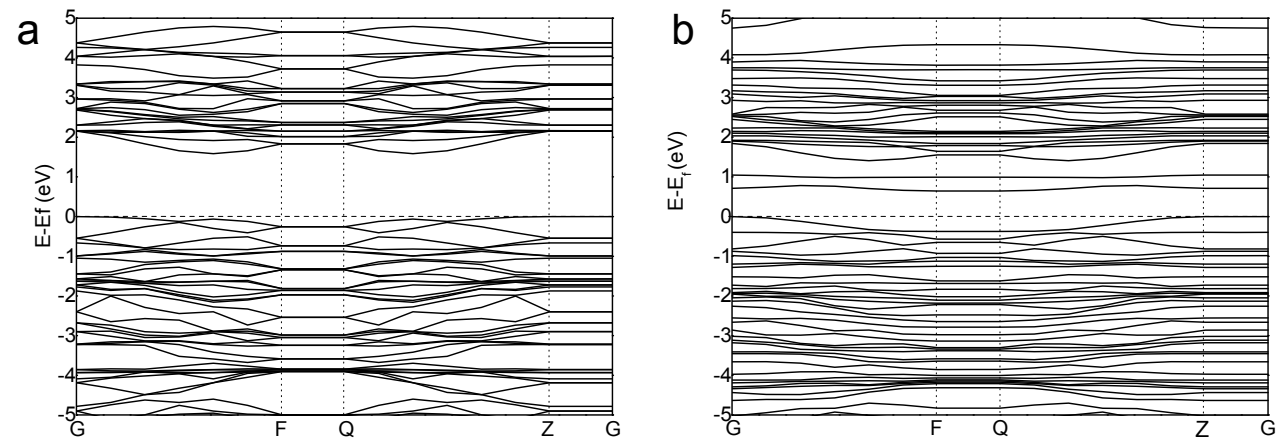

Figure S12. (a) Band structure of monolayer $2 \mathrm{H}-\mathrm{MoS}_{2}$. (b) Band structure of monolayer $\mathrm{MoS}_{2}$ with S-vacancies.

As shown in Figure S12, when S-vacancies are introduced, new bands appear in the gap near the Fermi level. These new gap states are responsible for hydrogen adsorption on the S-vacancies, which can decrease the hydrogen adsorption free energy of the S-vacancy sites on the basal plane and improve their HER activity.
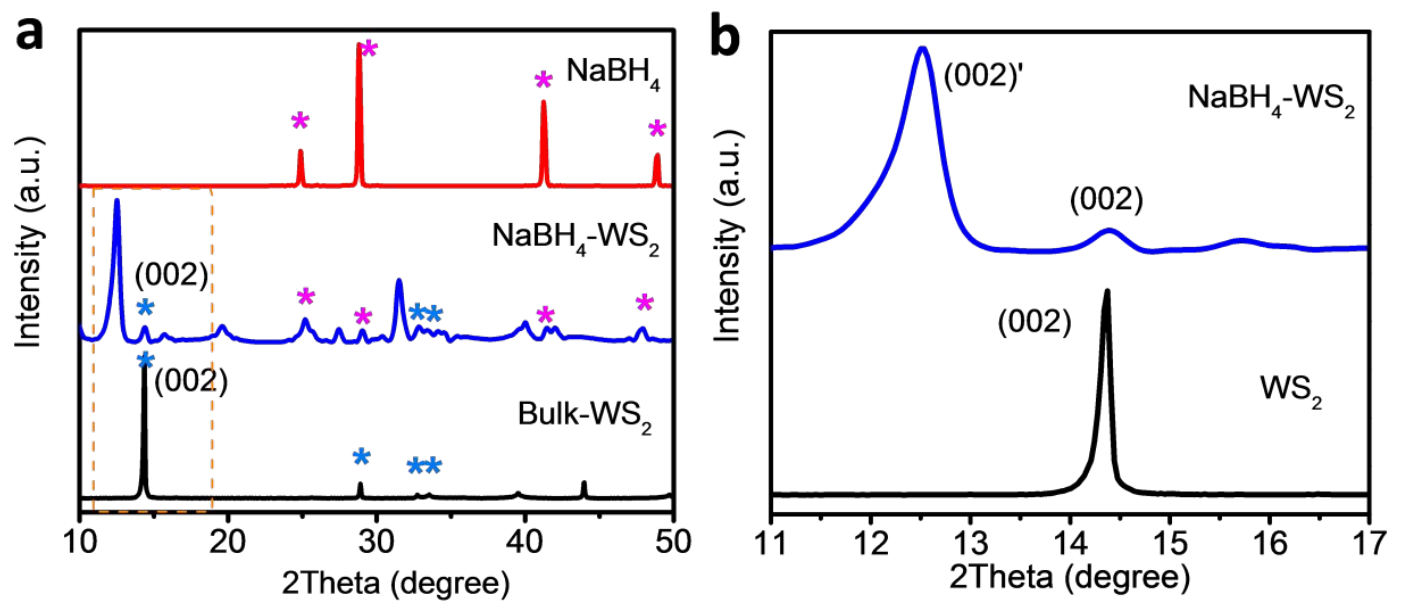

Figure S13. (a) XRD pattern of annealed bulk $\mathrm{WS}_{2}$ (black), $\mathrm{NaBH}_{4}$ (red) and their mixture (blue). (b) Enlargement of annealed bulk $\mathrm{WS}_{2}$ and $\mathrm{NaBH}_{4}-\mathrm{WS}_{2}$. 

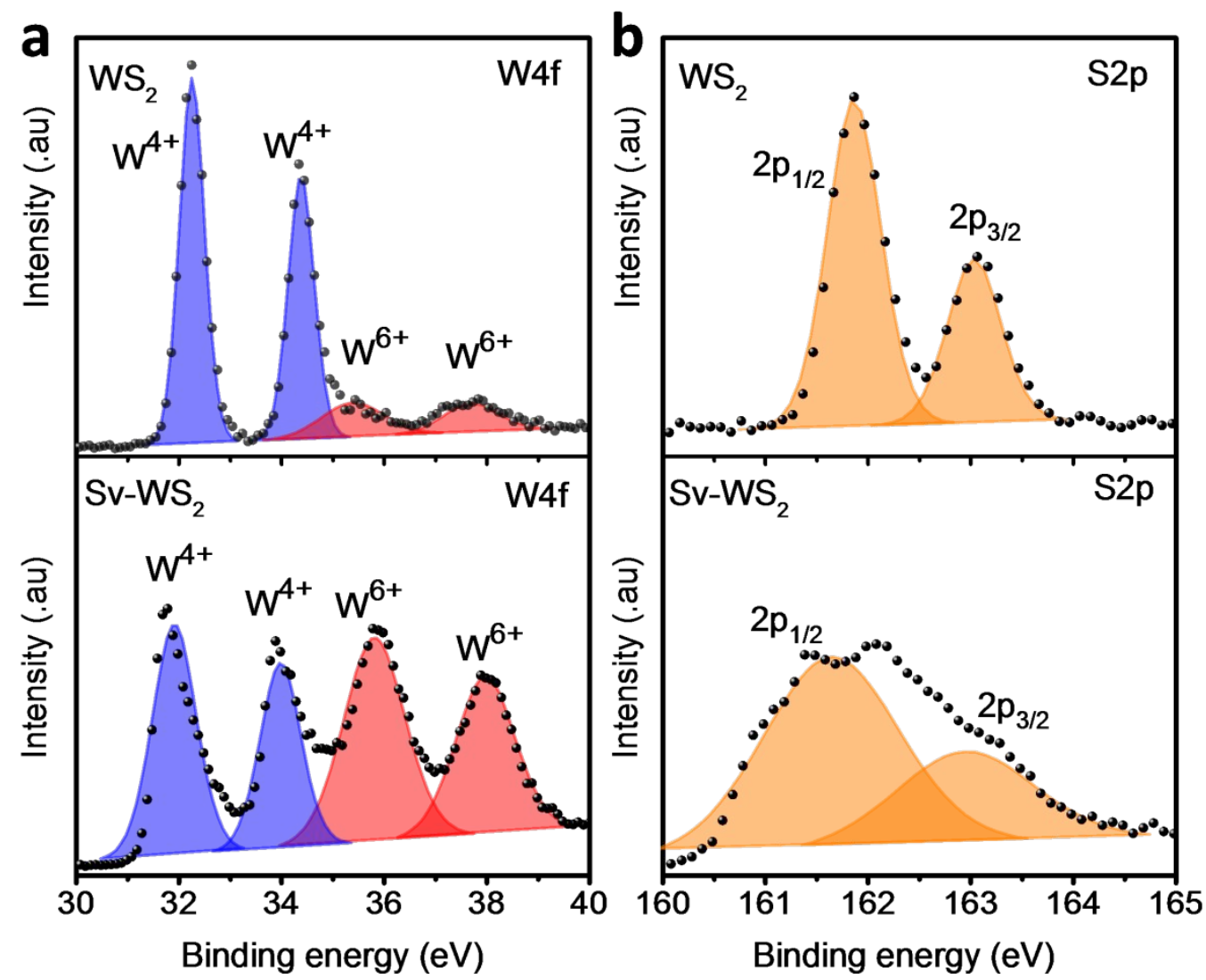

Figure S14. XPS spectra showing W $4 f(a), S 2 p(b)$ level peak regions for $W_{2}$ (up) and oxidized Sv-WS ${ }_{2}$ NSs (down).

For pristine $2 \mathrm{H}$ phase $2 \mathrm{D}-\mathrm{WS}_{2}, \mathrm{~W} 4 \mathrm{f}$ spectra consist of peaks around 33.2 and $31.1 \mathrm{eV}$ corresponding to the $\mathrm{W}^{4+} \mathrm{W}_{4} \mathrm{f}_{7 / 2}$ and $\mathrm{W}_{4} \mathrm{f}_{5 / 2}$ components, respectively. Notably, an obvious shift for both $\mathrm{W}_{4} \mathrm{f}_{7 / 2}$ and $\mathrm{W} 4 \mathrm{f}_{5 / 2}$ peaks to the higher binding energies at $\sim 35$ and $38 \mathrm{eV}$ was observed in Sv-WS 2 (Figure S14a, bottom), which is consistent with the valence state change of $\mathrm{W}^{6+}$. And this phenomenon is due to the easily oxidation of low valence state W exposed on surface of Sv$\mathrm{WS}_{2}$. Thus, the sulfur atom loss can be measured by normalizing $S 2 p$ peaks according to the total measured area ratio of all W $4 \mathrm{f}$ peaks between pristine $2 \mathrm{D}-\mathrm{WS}_{2}$ and $\mathrm{Sv}-\mathrm{WS}_{2}$. 


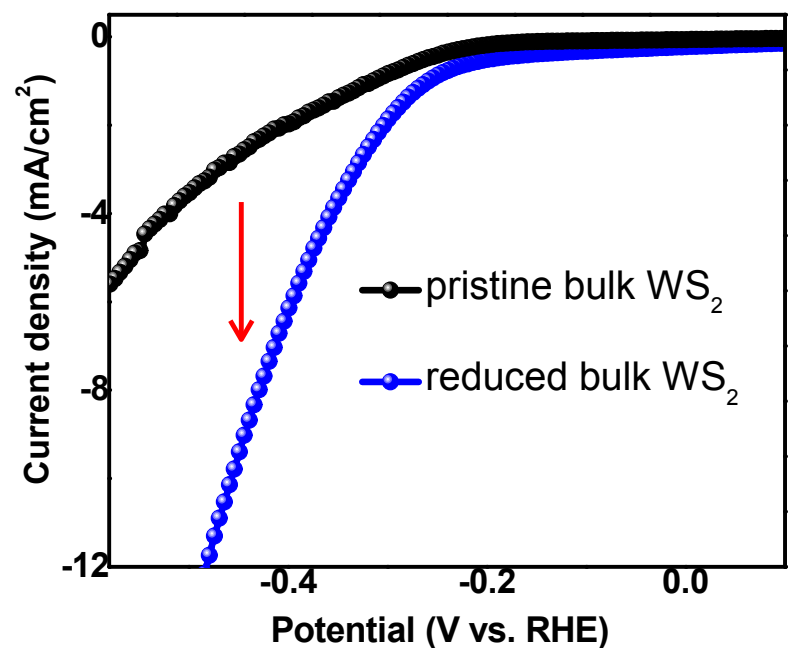

Figure S15. $J-V$ curves of pristine bulk-WS $S_{2}$ and $\mathrm{NaBH}_{4}$ treated bulk-WS 2 . 
Table S3. Summary of HER activities of $S v-W S_{2}$ and the reported $W_{2}$ materials in $0.5 \mathrm{M} \mathrm{H}_{2} \mathrm{SO}_{4}$.

\begin{tabular}{|c|c|c|c|c|}
\hline Sample & $\begin{array}{c}\text { Catalytic active } \\
\text { sites }\end{array}$ & $\eta_{-10^{a}}$ & $\begin{array}{l}\text { Tafel } \\
\text { slope } \\
\left.\text { V dec }{ }^{-1}\right]\end{array}$ & Ref \\
\hline Metallic $\mathrm{WS}_{2} \mathrm{NS}$ & $\begin{array}{l}\text { basal plane } \\
\text { and } \\
\text { edge sites }\end{array}$ & $\sim 240$ & 60 & [11] \\
\hline $\mathrm{WS}_{2} \mathrm{NS}$ & edge sites & $\sim 350$ & 91 & [12] \\
\hline $\mathrm{WS}_{2}$ Nanoribbons & edge sites & $\sim 260$ & 58 & [13] \\
\hline $\begin{array}{l}\text { WS2/grapheme } \\
\text { film }\end{array}$ & edge sites & 306 & 67 & [14] \\
\hline $\mathrm{W}_{18} \mathrm{O}_{49} @ W_{2}$ NRs & edge sites & 310 & 86 & {$[15]$} \\
\hline$S v-W S_{2}$ & S vacancies & 241 & 78 & $\begin{array}{l}\text { This } \\
\text { work }\end{array}$ \\
\hline
\end{tabular}

${ }^{a}$ n (vs. RHE) for $\mathrm{j}=-10 \mathrm{~mA} \mathrm{~cm}{ }^{-2}[\mathrm{mV}]$ 

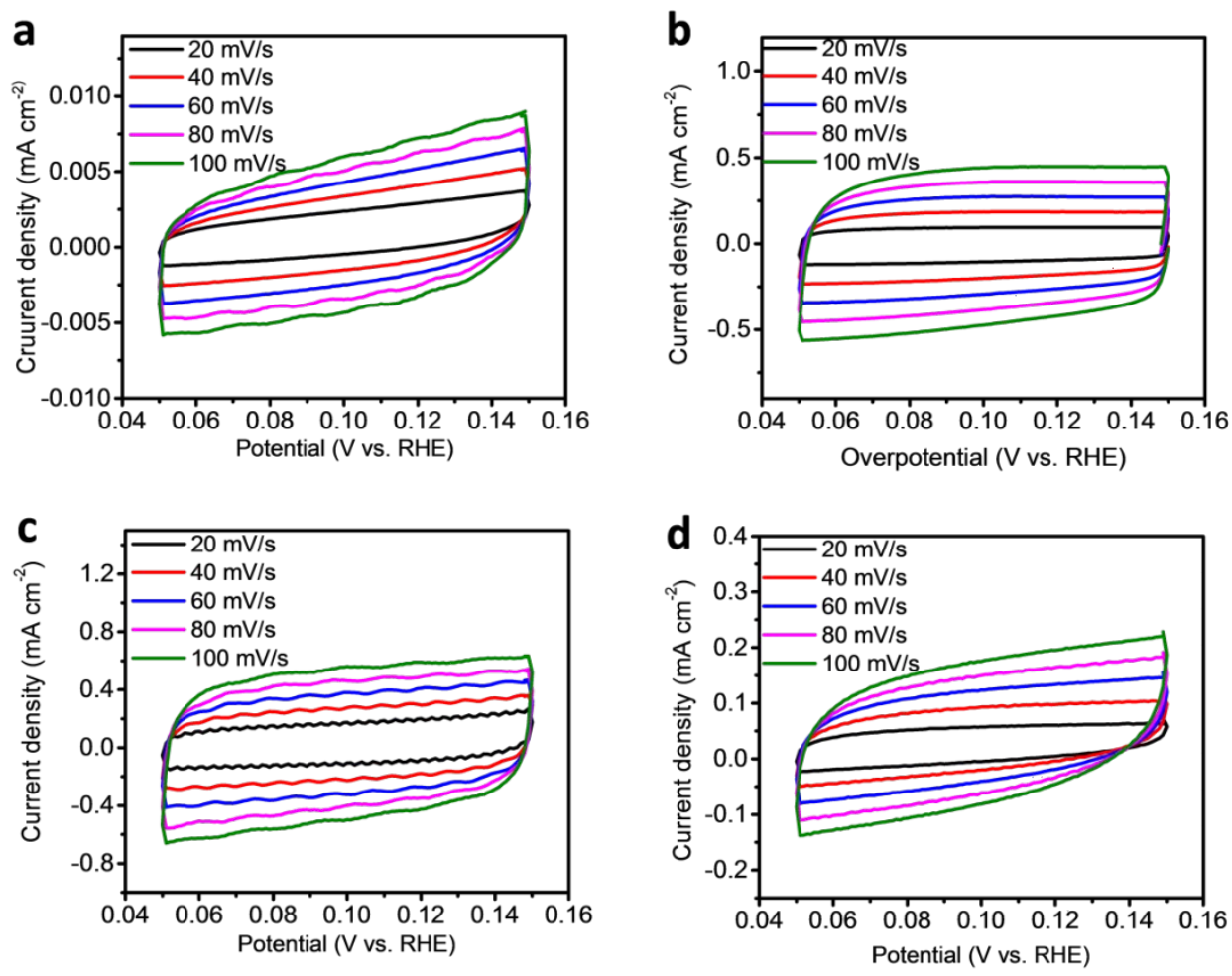

Figure S16. Cyclic voltammetry curves of (a) P-WS 2 , (b) $0.25-S v-W S_{2}$, (c) 0.35 Sv-WS 2 and (d) 0. 5-Sv-WS 2 NSs, respectively, under different scan rates, in the region of $0.1-0.2 \mathrm{~V} v s$. RHE. These data were used to caculate the electrochemical double-layer capacitance $\left(C_{d \mathrm{dl}}\right)$. Plots show the extraction of the $\mathrm{C}_{\mathrm{dl}}$ for different $\mathrm{MoS}_{2}$ samples shown in Figure $5 \mathrm{e}$ in the main text.

\section{References}

(1) Zak, A.; Feldman, Y.; Lyakhovitskaya, V.; Leitus, G.; R. Biro, P.; Wachtel, E.; Cohen, H.; Reich, S.; Tenne, R. Alkali Metal Intercalated Fullerene-Like $\mathrm{MS}_{2}(\mathrm{M}=\mathrm{W}, \mathrm{Mo})$ Nanoparticles and Their Properties. J. Am. Chem. Soc. 2002, 124, 4747-4758.

(2) Somoano, R. B.; Hadek, V.; Rembaum, A. J. Alkali Metal Intercalates of Molybdenum Disulfide. J. Chem. Phys. 1973, 58, 697-701. 
(3) Gong, Y.; Lei, S.; Ye, G.; Li, B.; He, Y.; Keyshar, K.; Zhang, X.; Wang, Q.; Lou, J.; Liu, Z.; Vajtai, R.; Zhou, W.; Ajayan, P. M. Two-Step Growth of Two-Dimensional WSe $/ \mathrm{MoSe}_{2}$ Heterostructures. Nano Lett. 2015, 15, $6135-6141$.

(4) Zheng, J.; Zhang, H.; Dong, S.; Liu, Y.; Tai Nai, C.; Suk Shin, H.; Young Jeong, H.; Liu, B.; Ping Loh, K. High Yield Exfoliation of Two-Dimensional Chalcogenides Using Sodium Naphthalenide. Nat. Commun. 2014, 5, 2995.

(5) Lee, J.; Kang, S.; Yim, K.; Kim, K. Y.; Jang, H. W.; Kang, Y.; Han, S. Hydrogen Evolution Reaction at Anion Vacancy of Two-Dimensional Transition-Metal Dichalcogenides: Ab Initio Computational Screening. J. Phy. Chem. Lett. 2018, 9, 2049-2055.

(6) Benck, J. D.; Hellstern, T. R.; Kibsgaard, J.; Chakthranont, P.; Jaramillo, T. F. Catalyzing the Hydrogen Evolution Reaction (HER) with Molybdenum Sulfide Nanomaterials. ACS Catal. 2014, 4, 3957-3971.

(7) Li, G.; Zhang, D.; Qiao, Q.; Yu, Y.; Peterson, D.; Zafar, A.; Kumar, R.; Curtarolo, S.; Hunte, F.; Shannon, S.; Zhu, Y.; Yang, W.; Cao, L. All The Catalytic Active Sites of $\mathrm{MoS}_{2}$ for Hydrogen Evolution. J. Am. Chem. Soc. 2016, 138, 16632-16638.

(8) Ye, G. L.; Gong, Y. J.; Lin, J. H.; Li, B.; He, Y. M.; Pantelides, S. T.; Zhou, W.; Vajtai, R.; Ajayan, P. M. Defects Engineered Monolayer $\mathrm{MoS}_{2}$ for Improved Hydrogen Evolution Reaction. Nano Lett. 2016, 16, 1097-1103. 
(9) Tsai, C.; Li, H.; Park, S.; Park, J.; Han, H. S.; Norskov, J. K.; Zheng, X. L.; Abild-Pedersen, F. Electrochemical Generation of Sulfur Vacancies in the Basal Plane of $\mathrm{MoS}_{2}$ for Hydrogen Evolution. Nat. Commun. 2017, 8, 15113.

$$
\text { Li, H.; Tsai, C.; Koh, A. L.; Cai, L.; Contryman, A. W.; Fragapane, A. H.; }
$$

Zhao, J.; Han, H. S.; Manoharan, H. C.; Abild-Pedersen, F.; Norskov, J. K.;

Zheng, X. Activating and Optimizing $\mathrm{MoS}_{2}$ Basal Planes for Hydrogen

Evolution Through the Formation of Strained Sulphur Vacancies. Nat. Mater. 2016, 15, 48-53.

(11) Voiry, D.; Yamaguchi, H.; Li, J.; Silva, R.; Alves, D. C. B.; Fujita, T.; Chen, M.; Asefa, T.; Shenoy, V. B.; Eda, G.; Chhowalla, M. Enhanced Catalytic Activity in Strained Chemically Exfoliated $\mathrm{WS}_{2}$ Nanosheets for Hydrogen Evolution. Nat. Mater. 2013, 12, 850-855.

$$
\text { Guo, X.; Ji, J.; Jiang, Q.; Zhang, L.; Ao, Z.; Fan, X.; Wang, S.; Li, Y.; }
$$

Zhang, F.; Zhang, G.; Peng, W. Few-Layered Trigonal WS 2 NanosheetCoated Graphite Foam as an Efficient Free-Standing Electrode for a Hydrogen Evolution Reaction. ACS Appl. Mater. 2017, 9, 30591-30598.

$$
\text { Lin, J.; Peng, Z.; Wang, G.; Zakhidov, D.; Larios, E.; Yacaman, M. J.; }
$$

Tour, J. M. Enhanced Electrocatalysis for Hydrogen Evolution Reactions from $\mathrm{WS}_{2}$ Nanoribbons. Adv. Energy Mater. 2014, 4, 1301875.

$$
\text { Pu, Z.; Liu, Q.; Asiri, A. M.; Obaid, A. Y.; Sun, X. One-Step }
$$

Electrodeposition Fabrication of Graphene Film-Confined $\mathrm{WS}_{2}$ 
Nanoparticles with Enhanced Electrochemical Catalytic Activity for Hydrogen Evolution. Electrochim. Acta 2014, 134, 8.

(15) Wang, X.; Gan, X.; Hu, T.; Fujisawa, K.; Lei, Y.; Lin, Z.; Xu, B.; Huang, Z. H.; Kang, F.; Terrones, M.; Lv, R. Noble-Metal-Free Hybrid Membranes for Highly Efficient Hydrogen Evolution. Adv. Mater. 2017, 29, 1603617. 